\title{
SIR-SI Mathematical Model for Zika Virus Progression Dynamics in India: A Case Study
}

\author{
$\underline{\text { Ravins Dohare', Manoj Kumar }}$, Shweta Sankhwar ${ }^{3}$, Narender Kumar ${ }^{4}$, \\ $\underline{\text { Surendra Kumar Sagar }}^{5}$, Jugal Kishore ${ }^{6}$
}

${ }^{1}$ Centre for Interdisciplinary Research in Basic Sciences, Jamia Millia Islamia, Jamia Nagar, New Delhi, India.

${ }^{2}$ Centre for Economic Studies and Planning, Jawaharlal Nehru University, India.

${ }^{3}$ Department of Computer Science, Maitreyi College (University of Delhi), New Delhi, India.

${ }^{4}$ Department of Mathematics, Gargi College, University of Delhi, India.

${ }^{5}$ Department of Zoology, Swami Shraddhanand College (University of Delhi), Delhi, India.

${ }^{6}$ Department-Community Medicine, VMMC \& Safdarjung Hospital (Ministry of Health \& Family Welfare) New Delhi, India.

DOI: https://doi.org/10.24321/0019.5138.202132

I $\mathbf{N} \quad \mathbf{F} \mathbf{O}$

\section{Corresponding Author:}

Surendra Kumar Sagar, Department of Zoology, Swami Shraddhanand College (University of Delhi), Delhi, India.

E-mail Id:

ssagar.du@yahoo.com

Orcid Id:

https://orcid.org/0000-0003-4256-5475

How to cite this article:

Dohare R, Kumar M, Sankhwar S, Kumar N, Sagar SK, Kishore J. SIR-SI Mathematical Model for Zika Virus Progression Dynamics in India: A Case Study. J Commun Dis. 2021; 53(2): 100-104.

Date of Submission: 2021-05-19

Date of Acceptance: 2021-06-28

\section{$\begin{array}{llllllll}\mathbf{A} & \mathbf{B} & \mathbf{S} & \mathbf{T} & \mathbf{R} & \mathbf{A} & \mathbf{C} & \mathbf{T}\end{array}$}

Viral diseases are very hazardous for humanity because in the case of most viral diseases, drugs are not effective. At present, the whole world is living with the fear of COVID-19. From time to time, several viral diseases have been troubling human life. In this article, we have tried to capture the progression dynamics of Zika Virus (ZIKV) infection in the Indian scenario. A constructed model is based on compartment modelling. In the model, Susceptible-Infected-Recovered (SIR) structure is used for the human population and Susceptible-Infected (SI) structure is used for mosquito population. The value of the basic reproduction number $\left(R_{0}\right)$ is computed 0.36 at baseline values of parameters involved in the model. The lower value of $R_{0}$ suggests that infection was unable to spread in the human population. Sensitive analysis for $R_{0}$ revealed that the most accountable parameter in the spread of infection was mosquito biting rate. The modelling technique might be useful for other diseases also.

Keywords: Zika Virus Infection, Disease-free Equilibrium, Basic Reproduction Number, Numerical Simulation

\section{Introduction}

Zika virus is an emerging mosquito-borne Flavivirus in the Flaviviridae family that is responsible for many recent epidemics in America and the Caribbean region. ${ }^{1}$ Zika virus (ZIKV) can be transmitted via mosquito bites, sexual contact, and blood transfer (very likely but not confirmed) from a pregnant woman to her foetus. Aedes mosquito was found to be the main cause of transmission of ZIKV infection in humans. This transmission is bidirectional i.e. infected mosquitoes infect humans and vice-versa. ${ }^{1,2}$ Transmission by other means is very low as compared to transmission by mosquitoes. ${ }^{2}$ The common symptoms of ZIKV infection are fever, headache, joint pain, muscle pain, rashes, red eyes ${ }^{3}$ etc. As Zika may be transmitted vertically, in pregnancy cases, ZIKV infection may cause microcephaly or other serious brain defects. ${ }^{4}$ Further ZIKV infection may be responsible for other problems like miscarriage, stillbirth, and other birth defects. 
In human (or animal) population, mathematical modelling is used as a powerful tool in understanding and analysing the dynamics of the infection. Although, we cannot capture the dynamics of infection perfectly by mathematical modelling since the conversion of a natural problem into a mathematical framework depends on many assumptions and parameters. There are different techniques in the mathematical modelling for infection dynamics. Compartment modelling has its novelty in these techniques. Many previous studies ${ }^{2,5-8}$ have been done on this approach for infectious diseases.

The Ministry of Health and Family Welfare of India (MoHFW) initially reported three cases of ZIKV infection in Bapunagar area of Ahmedabad District, Gujarat (India) on 15 May 2017. Later on, these three cases viz. (i) 34-year-old female (delivered a clinically healthy baby) (ii) 22-year-old pregnant female, and (iii) 64-year-old male, were confirmed to be infected with ZIKV. According to the WHO assessment, it was the first case of ZIKV infection in India. Although, findings support the fact that the transmission level of infection was very low. Fortunately, after these cases, there are no other confirmed cases anywhere in India. ${ }^{9}$

\section{Materials and Methods}

\section{Mathematical Formulation}

The mathematical model has always been constructed under certain constraints since mathematical formulations, unlike real situations, are based on perfect criteria whereas a number of minor reluctant parameters may affect the scenario of physical problems. The assumptions under which the model is constructed are as follows:

- The total population of hosts is always constant i.e. $\mathrm{N}_{h}=\mathrm{S}_{h}+\mathrm{I}_{\mathrm{h}}+\mathrm{R}_{\mathrm{h}}$ and the total population of mosquitoes is also constant i.e. $\mathrm{N}_{m}=\mathrm{S}_{m}+I_{m}$.

- There is no direct transmission among humans.

- There is no direct transmission among mosquitoes.

- There is no direct recovery for susceptible humans.

By ignoring a lot of parameters and constraints, the model can be written in mathematical framework as follows.

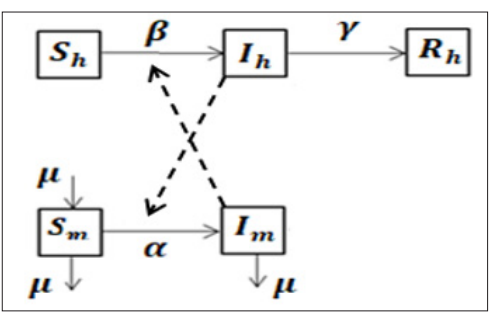

Figure I.Systematic Representation of Mathematical Model

$$
\begin{aligned}
& \frac{d S_{h}}{d t}=-\beta S_{h} I_{m} \\
& \frac{d I_{h}}{d t}=\beta S_{h} I_{m}-\gamma I_{h} \\
& \frac{d R_{h}}{d t}=\gamma I_{h} \\
& \frac{d S_{m}}{d t}=\mu N_{m}-\alpha S_{m} I_{h}-\mu S_{m} \\
& \frac{d I_{m}}{d t}=\alpha S_{m} I_{h}-\mu I_{m}
\end{aligned}
$$

Where $\beta$ is the rate at which susceptible human population becomes infectious, $\gamma$ is the rate at which the infectious human population recovered, and is the rate at which susceptible mosquito population becomes infected. We used previous studies to simulate the values of these parameters. The value of mosquito biting rate per human per day $\left(b_{m}\right)$, mosquito death rate $(\mu)$ average ratio of mosquito to human $\left(a_{m}\right)$, transmission probability from infectious mosquitoes to susceptible humans $\left(p_{m h}\right)$, and transmission probability from infectious humans to susceptible mosquitoes $\left(p_{h m}\right)$ are obtained from previous studies. ${ }^{6-9}$ The infection rate in human population $(\beta)$ is the product of $b_{m}, p_{m h^{\prime}}$ and $a_{m}$ i.e. $\beta=b_{m} \times p_{m h} \times a_{m}$. On the

\begin{tabular}{|c|c|c|c|c|}
\hline Notation & Description & Range & Baseline value & Reference \\
\hline$b_{m}$ & Mosquito biting rate (per mosquito per day) & $0.3-1$ & 0.6 & {$[6]$} \\
\hline $\mathrm{p}_{\mathrm{mh}}$ & $\begin{array}{l}\text { Transmission probability from infectious mosquito to } \\
\text { susceptible human per bite }\end{array}$ & $0.1-0.75$ & 0.1 & {$[6]$} \\
\hline $\mathrm{p}_{\mathrm{hm}}$ & $\begin{array}{l}\text { Transmission probability from infected human to } \\
\text { susceptible mosquito per bite }\end{array}$ & $0.3-0.75$ & 0.3 & {$[6]$} \\
\hline$\gamma$ & $\begin{array}{l}\text { Rate from infected human population to recovered human } \\
\text { population }\end{array}$ & $0.035-0.071$ & 0.05 & {$[11],[12]$} \\
\hline$\mu$ & Mosquitos death rate (per day) & $4-35$ & 10 & {$[6],[8]$} \\
\hline$a_{m}$ & Average ratio of mosquitos to human & $1-10$ & 6 & [7] \\
\hline
\end{tabular}
other hand, the infection rate in mosquito population $(\alpha)$ is equal to $b_{m} \times p_{h m}$. Range and baseline values of all these parameters are given in Table 1.

Table I.Parameters description used in model with range and baseline values 
Disease Free Equilibrium: The equilibrium point of the system of equations (1-5) at $\left(\mathrm{S}_{\mathrm{h}}{ }^{*}, \mathrm{I}_{\mathrm{h}}{ }^{*}, \mathrm{R}_{\mathrm{h}}{ }^{*}, \mathrm{~S}_{\mathrm{m}}{ }^{*}, \mathrm{I}_{\mathrm{m}}{ }^{*}\right)$ is the simultaneous solution for the following system of equations.

$-\beta S_{\mathrm{h}}^{*} I_{\mathrm{m}}^{*}=0$

$\beta \mathrm{S}_{\mathrm{h}}^{*} \mathrm{I}_{\mathrm{m}}^{*}-\gamma \mathrm{I}_{\mathrm{h}}^{*}=0$

$\gamma \mathrm{I}_{\mathrm{h}}^{*}=0$

$\mu \mathrm{I}_{\mathrm{m}}-\alpha \mathrm{S}_{\mathrm{m}}^{*} \mathrm{I}_{\mathrm{h}}^{*}=0$

$\alpha \mathrm{S}_{\mathrm{m}}^{*} \mathrm{I}_{\mathrm{h}}^{*}-\mu \mathrm{I}_{\mathrm{m}}=0$

Using the relationship $\mathrm{N}_{\mathrm{h}}=\mathrm{S}_{\mathrm{h}}{ }^{*}+\mathrm{I}_{\mathrm{h}}{ }^{*}+\mathrm{R}_{\mathrm{h}} *$ and $\mathrm{N}_{\mathrm{m}}=\mathrm{S}_{\mathrm{m}}{ }^{*}+\mathrm{I}_{\mathrm{m}}$ * the system of equations (1a-5a) will reduce to the following system of equations:

$-\beta \mathrm{S}_{\mathrm{h}}^{*} \mathrm{I}_{\mathrm{m}}^{*}=0$

$\beta \mathrm{S}_{\mathrm{h}}^{*} \mathrm{I}_{\mathrm{m}}^{*}-\gamma \mathrm{I}_{\mathrm{h}}^{*}=0$

$\mu\left(S_{m}+I_{m}\right)-\alpha S_{m}^{*} I_{h}^{*}=0$

After solving this system of equations (8-10), two equilibrium points of model $(1-5)$ are $(0,0,0,0,0)$ and $\left(\mathrm{S}_{\mathrm{h}}{ }^{*}, 0, \mathrm{~N}_{\mathrm{h}}-\mathrm{S}_{\mathrm{h}}\right.$, $\mathrm{N}_{\mathrm{m}}, 0$ ).

Theorem 1: Let $f_{1}, f_{2}, f_{3}, f_{4^{\prime}}, f_{5}$ are the functions of $S_{h}, I_{h}, R_{h}$, $\mathrm{S}_{\mathrm{m}}, \mathrm{I}_{\mathrm{m}}$ such that.

$$
\begin{aligned}
& \mathrm{f}_{1} \equiv \frac{\mathrm{dS}}{\mathrm{dt}}=-\beta \mathrm{S}_{\mathrm{h}} ; \mathrm{S}_{\mathrm{h}}\left(\mathrm{t}_{0}\right)=\mathrm{S}_{\mathrm{h}_{0}} \\
& \mathrm{f}_{2} \equiv \frac{\mathrm{dI}}{\mathrm{dt}}=\beta \mathrm{S}_{\mathrm{h}} \mathrm{I}_{\mathrm{m}}-\gamma \mathrm{I}_{\mathrm{h}} ; \mathrm{I}_{\mathrm{h}}\left(\mathrm{t}_{0}\right)=\mathrm{I}_{\mathrm{h}_{0}} \\
& \mathrm{f}_{3} \equiv \frac{\mathrm{d \textrm {R } _ { \mathrm { h } }}}{\mathrm{dt}}=\gamma \mathrm{I}_{\mathrm{h}} ; \mathrm{R}_{\mathrm{h}}\left(\mathrm{t}_{0}\right)=\mathrm{R}_{\mathrm{h}_{0}} \\
& f_{4} \equiv \frac{d S_{m}}{d t}=\mu N_{m}-\alpha S_{m} I_{h} ; S_{m}\left(t_{0}\right)=S_{m_{0}} \\
& f_{5} \equiv \frac{d I_{m}}{d t}=\alpha S_{m} I_{h} ; I_{m}\left(t_{0}\right)=I_{m_{0}}
\end{aligned}
$$

Let $=\left\{\left(S_{h}, I_{h}, R_{h}, S_{m}, I_{m}\right):\left|S_{h}-S_{h 0}\right| \leq \delta_{1^{\prime}}, I_{h}-I_{h 0}\left|\leq \delta_{2^{\prime}},\right| R_{h}-R_{h 0} \mid \leq \delta_{3^{\prime}}\right.$ $\left.\left|S_{m}-S_{m 0}\right| \leq \delta_{4^{\prime}},\left|I_{m}^{-1} I_{m 0}\right| \leq \delta_{5}\right\}$, then the system of equations $(1 \mathrm{c}-5 \mathrm{c})$ has a unique solution.

Proof: All derivatives $\left|\frac{\partial f_{i}}{\partial x_{j}}\right| ; i, j=1,2,3,4,5$ with $x_{1}=S_{h}, x_{2}=I_{h}$, $x_{3}=R_{h}, x_{4}=S_{m}, x_{5}=I_{m}$ at DFE are continuous and bounded. Therefore by Derrick and Crrossman ${ }^{11}$ the system of equations $(5 a-5 c)$ has a unique solution. Consequently, the model represented by the system of equations (1-5) is well-posed and epidemiologically feasible.

\section{Basic Reproduction Number}

Reproduction number $\left(R_{0}\right)$ is the expected number of secondary cases generated by a single infection while the complete population is treated as susceptible. ${ }^{10}$ At diseasefree point, $I_{h}=R_{h}=0$, implies $S h=N_{h}$ and $I_{m}=0$ further implying $\mathrm{S}_{\mathrm{m}}=\mathrm{N}_{\mathrm{m}}$. Therefore, to compute basic reproduction, the following linearised subsystem associated with infectious classes is considered.

$\frac{d I_{h}}{d t}=\beta S_{h} I_{m}-\gamma I_{h}$

$\frac{d I_{m}}{d t}=\alpha S_{m} I_{h}-\mu I_{m}$

System of equations (11a-11b) can be rewritten in scaled variables such that:

$$
\begin{aligned}
& \frac{d i_{h}}{d t}=\beta s_{h} i_{m}-\gamma i_{h} \\
& \frac{d i_{m}}{d t}=\alpha s_{m} i_{h}-\mu i_{m}
\end{aligned}
$$

Where $s_{h}=\frac{s_{h}}{N_{h}}, i_{h}=\frac{I_{h}}{N_{h}}, r_{h}=\frac{R_{h}}{N_{h}}, s_{m}=\frac{s_{m}}{N_{m}}$ and $i_{m}=\frac{I_{m}}{N_{m}}$ which gives $N_{h}=N_{m}=1$. Let $X=\left(i_{h}, i_{m}^{h}\right)^{t}$ then linearised subsystem (12a-12b) $c$ can be written in matrix form as:

$\frac{d X}{d t}=(F+V) X$

Where $F$ (transmission matrix) and $V$ (Transition matrix) are defined in the following manner:

$F=\left(\begin{array}{cc}0 & \beta \\ \alpha & 0\end{array}\right), V=\left(\begin{array}{cc}-\gamma & 0 \\ 0 & -\mu\end{array}\right)$

We compute next generation matrix, $F=\left(\begin{array}{cc}0 & \beta \\ \alpha & 0\end{array}\right), V=\left(\begin{array}{cc}-\gamma & 0 \\ 0 & -\mu\end{array}\right)$.

The set of eigenvalues of matrix $G$ is $\left\{\sqrt{\frac{\alpha \beta}{\mu \nu}},-\sqrt{\frac{\alpha \beta}{\mu v}}\right\}$. Therefore basic reproduction number $\left(R_{0}\right)=\rho(G)=\sqrt{\frac{\alpha \beta}{\mu \nu}}$, where $\rho$ is the spectral radius of matrix $G$.

\section{Results and Discussion}

The basic reproduction number ${ }^{10}\left(\boldsymbol{R}_{d}\right)$ is a crucial parameter. Its value is treated as the threshold for infection spreading in the population. If the value of $\boldsymbol{R}_{0}>\boldsymbol{1}$ then the infection will invade the population, else it will not invade. The normalised sensitivity index $(\boldsymbol{\eta})$ of $\mathbf{R}_{\mathbf{0}}$ with respect to a parameter $\mathrm{x}$ is given by: $\eta_{x}^{R_{0}}=\frac{\partial R_{0}}{\partial x} \times \frac{x}{R_{0}}$

The value of sensitivity indexes of $R_{0}$ with respect to different parameters is given in Table 2. The magnitude of index reflects the relative importance of that variable to $R_{0}$. The sign of index represents the increase (in case of +ve sign) or decrease (in case of -ve sign) in $R_{0}$ with respect to the associated variables. The value of indexes suggest that $R_{0}$ is the most sensitive to mosquito biting rate $\left(b_{m}\right)$ whereas 
other parameters viz. average ratio of mosquito $\left(a_{m}\right)$ transmission probabilities $\left(p_{m h}\right.$ and $p_{h m}$ ), mosquito death rate $(\mu)$, and infection recovery rate $(\gamma)$ equally influence $R_{0}$. Moreover, mosquito death rate $(\mu)$ and infection recovery rate $(\gamma)$ have a negative relationship with $R_{0}$.

The relationship between basic reproduction number $\left(R_{0}\right)$ and transmission probability of infection from human to mosquito $\left(p_{h m}\right)$ at different mosquito biting rates $\left(b_{m}\right)$ is shown in Figure 2 . The figure suggests that $R_{0}$ increases with the value of $p_{h m}$ and this value is higher at a high mosquito biting rate. Although, $R_{0}$ could not reach unity for a given range of parameters therefore it is concluded that the disease cannot propagate in the population at the given parameter values. The relationship between $R_{0}$ and infection recovery rate $(\gamma)$ at different values of mosquito biting rate $\left(\mathrm{b}_{\mathrm{m}}\right)$ is shown in Figure 3 . This figure depicts that $R_{0}$ is a decreasing function of infection recovery rate $(\gamma)$.

Table 2.Sensitivity Index for Basic Reproduction Number with respect to different Parameters

\begin{tabular}{|c|c|c|c|c|c|c|}
\hline Parameters & $\mathrm{b}_{\mathrm{m}}$ & $\mathrm{a}_{\mathrm{m}}$ & $\mathrm{p}_{\mathrm{mh}}$ & $\mathrm{p}_{\mathrm{hm}}$ & $\mu$ & $\gamma$ \\
\hline Sensitivity index & 1 & 0.5 & 0.5 & 0.5 & -0.5 & -0.5 \\
\hline
\end{tabular}

According to the results, mosquito biting rate is the most influential parameter to prevent the infection rate. In the report on ZIKV infection in India, WHO also suggested controlling the contact between mosquitoes and humans, and also advised to control the mosquito breeding points to prevent ZIKV infection. ${ }^{9}$ WHO also recommended taking precautions while travelling to high-risk areas, especially for pregnant women. These precautions from mosquito bites include repellents, creams, coils, mosquito nets, longsleeved dresses and properly fitted rooms. WHO had also advised safer sexual practices to reduce the risk of ZIKV infection. However, WHO did not recommend any type of restriction on travelling or trading in India.

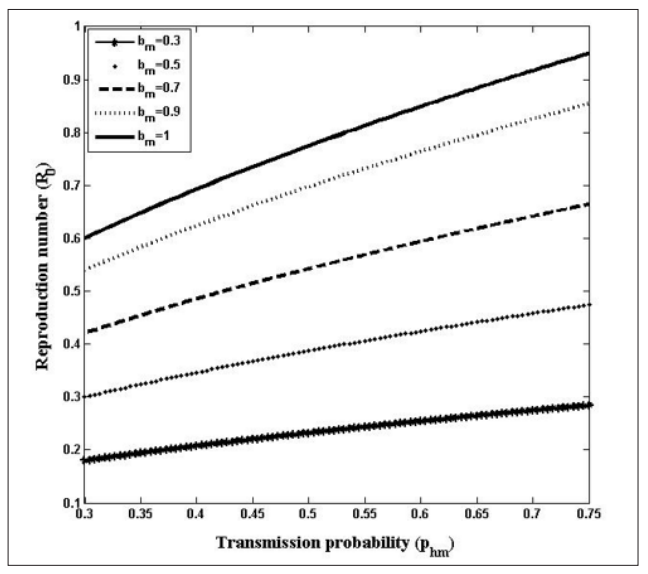

Figure 2.Relation between $\mathbf{R}_{0}$ and Transmission Probability of Infection from Human to Mosquito $\left(p_{h m}\right)$ at different values of Mosquito Biting Rate

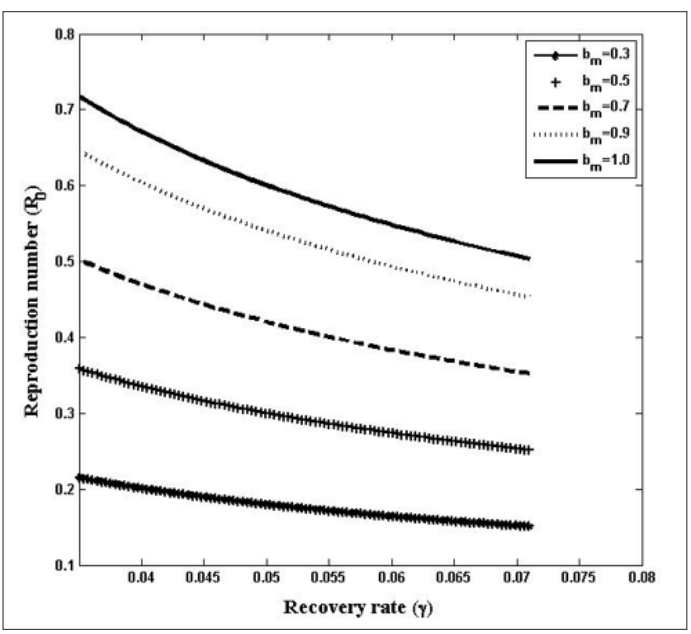

Figure 3.Relation between $\mathbf{R}_{\mathbf{0}}$ and Human Infection Recovery Rate $(\gamma)$ at different values of Mosquito Biting Rate

\section{Conclusion}

We constructed a mathematical model using compartment modelling to investigate the dynamics of ZIKV infection. The values of used parameters in the model are taken in the context of the Indian climate. The lower value of the threshold parameter $R_{0}=0.36$ computed at baseline values depict that ZIKV infection could not propagate in the population. This study supports the WHO report for ZIKV infection in the Indian scenario.

\section{Acknowledgement}

This work is partially supported by the Science and Engineering Research Board, D.S.T., Govt. of India, India under Grant No. EEQ/2016/000509.

\section{Source of Funding: None}

\section{Conflict of Interest: None}

\section{References}

1. Pan American Health Organization/ World Health Organization [Internet]. Zika; 2016 Apr 6[cited 2017 Nov 30]. Available from: http://www.paho.org/hq/ index.php?option=com_content $\&$ view=article \&id=1 $1585 \% 3$ Azika-virus-infection \&catid=8424\%3Aconten ts\&ltemid=41688\&lang=en

2. Cauchemez S, Besnard M, Bompard $P$, Dub $T$, Guillemette-Artur P, Eyrolle-Guignot D, Salje H, Van Kerkhove MD, Abadie V, Garel C, Fontanet A, Mallet HP. Association between Zika virus and microcephaly in French Polynesia, 2013-15: a retrospective study. Lancet. 2016;387(10033):2125-32. [Pubmed] [Google Scholar]

3. Centers for Disease Control and Prevention [Internet]. About Zika; 2014 Nov 5 [cited 2017 Sep 28]. Available from: https://www.cdc.gov/zika/about/index.html

4. Cao-Lormeau VM, Blake A, Mons S, Lastère S, Roche C, 
Vanhomwegen J, Dub T, Baudouin L, Teissier A, Larre $P$, Vial AL, Decam C, Choumet V, Halstead SK, Willison HJ, Musset L, Manuguerra JC, Despres P, Fournier E, Mallet HP, Musso D, Fontanet A, Neil J, Ghawché F. Guillain-Barré Syndrome outbreak associated with Zika virus infection in French Polynesia: a case-control study. Lancet. 2016;387(10027):1531-9. [PubMed] [Google Scholar]

5. Kumar N, Abdullah M, Faizan MI, Ahmed A, Alsenaidy HA, Dohare R, Parveen S. Progression dynamics of Zika fever outbreak in El Salvador during 2015-2016: a mathematical modeling approach. Future Virol. 2017;12(5):271-81. [Google Scholar]

6. Andraud M, Hens N, Marais C, Beutels P. Dynamic Epidemiological Models for Dengue Transmission: $A$ Systematic Review of Structural Approaches. PLoS One. 2012;7(11):e49085. [PubMed] [Google Scholar]

7. Medeiros LC, Castilho CA, Braga C, de Souza WV, Regis $\mathrm{L}$, Monteiro AM. Modeling the Dynamic Transmission of Dengue Fever: Investigating Disease Persistence. PLoS Negl Trop Dis. 2011;5(1):e942. [PubMed] [Google Scholar]

8. Chikaki E, Ishikawa H. A dengue transmission model in Thailand considering sequential infections with all four serotypes. J Infect Dev Ctries. 2009;3(9):711-22. [PubMed] [Google Scholar]

9. World Health Organization [Internet]. Zika virus infection - India; [cited 2021 Jul 08]. Available from: https://www.who.int/news-room/fact-sheets/detail/ zika-virus

10. Diekmann O, Heesterbeek JA, Metz JA. On the definition and the computation of the basic reproduction ratio Ro in models for infectious diseases in heterogeneous populations. J Math Biol. 1990;28(4):365-82. [PubMed] [Google Scholar]

11. Derrick, N.R. and Grossman, S.L. Differential Equation with applications. Addison Wesley Publishing Company, Inc. Philippines (1976). 